\title{
Marked Enteropathy in an Accelerated Macaque Model of AIDS
}

\author{
Joshua D. Croteau, Elizabeth L. Engle, Suzanne E. Queen, Erin N. Shirk, and M. Christine Zink
}

From the Department of Molecular and Comparative Pathobiology, Johns Hopkins University School of Medicine, Baltimore, Maryland

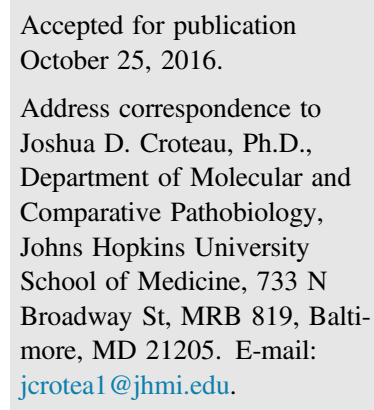

\begin{abstract}
Enteropathy in HIV infection is not eliminated with combination antiretroviral therapy and is possibly linked to microbial translocation. We used a rapidly progressing SIV/pigtailed macaque model of HIV to examine enteropathy and microbial translocation. Histologic evidence of intestinal disease was observed in only half of infected macaques during late-stage infection (LSI). Combination antiretroviral therapy initiated during acute infection prevented intestinal disease. In the ileum and colon, enteropathy was associated with increased caspase- 3 staining, decreased $\mathrm{CD}^{+}{ }^{+}$T cells, and increased SIVinfected cells. $\mathrm{CD}^{+} \mathrm{T}$ cells were preserved in LSI animals without intestinal disease, and levels of CD3 staining in all LSI animals strongly correlated with the number of infected cells in the intestine and plasma viral load. Unexpectedly, there was little evidence of microbial translocation as measured by soluble CD14, soluble CD163, lipopolysaccharide binding protein, and microbial 16s ribosomal DNA. Loss of epithelial integrity indicated by loss of the tight junction protein claudin-3 was not observed during acute infection despite significantly fewer T cells. Claudin-3 was reduced in LSI animals with severe intestinal disease but did not correlate with increased microbial translocation. LSI animals that did not develop intestinal disease had increased T-cell intracytoplasmic antigen 1-positive cytotoxic T lymphocytes, suggesting a robust adaptive cytotoxic T-lymphocyte response may, in part, confer resilience to SIV-induced intestinal damage. (Am J Pathol 2017, 187: 589-604; http://dx.doi.org/ 10.1016/j.ajpath.2016.10.019)
\end{abstract}

With the implementation of combination antiretroviral therapy (cART) HIV-infected people are living longer, healthier lives. The number and median age of people living with HIV has increased rapidly since the era before cART with $>37$ million individuals infected worldwide. ${ }^{1}$ As this population grows and ages, management of chronic HIVassociated conditions is becoming of increasing importance in the health community.

More than half of all HIV-infected patients experience gastrointestinal (GI) symptoms such as pain and diarrhea. In addition, nearly all patients on long-term cART exhibit GI complications at some point. ${ }^{2}$ These complications have been evident since the beginning of the AIDS epidemic and are often multifaceted, including gastritis, enteropathy, and upper and/or lower GI opportunistic infection with viral, microbial, and protozoal organisms. ${ }^{3}$ Visual evaluation of hematoxylin and eosin-stained intestinal sections is sufficient to make a diagnosis of HIV-associated GI disease. In addition, differential diagnoses may be made implicating specific pathogens contributing to overall disease with the use of a variety of techniques such as PCR, immunohistochemistry (IHC), and in situ hybridization (ISH). ${ }^{4}$

The pathologic changes that occur in the gut of HIVinfected individuals, both cART treated and untreated, are thought to result from complex HIV-induced immunologic changes. $\mathrm{CD}^{+} \mathrm{T}$ cells are rapidly and dramatically decreased in the GI tract of acutely and chronically HIVinfected individuals and are often reported to recover incompletely with implementation of cART. ${ }^{5,6}$ Some specialized lineages of $\mathrm{T}$ cells, including $\mathrm{T}$ helper type 17 cells and $\mathrm{T}$ regulatory cells, are affected by this loss,

\footnotetext{
Supported by NIH grants R01 MH087233 (M.C.Z.), R01 MH085554 (M.C.Z.), P01 MH070306, P30 MH075673, and P40 OD013117. Antiretroviral drugs for macaques were donated by Gilead Sciences (tenofovir), Bristol-Myers Squibb (atazanavir), Merck (integrase inhibitor L000870812/ INSTI), and Hoffmann La Roche (saquinavir).

Disclosures: None declared.
} 
contributing to the inflammatory imbalance. ${ }^{7}$ Macrophages, which are a large constituent of the gut and typically of an anti-inflammatory profile, are infected early on. However, they persist and even accumulate concurrently with the loss of $\mathrm{CD}^{+}{ }^{+}$T cells. ${ }^{8,9}$ Dendritic cells (DCs), which assume an antigen-presenting role in the gut, are rapidly lost with infection and are replaced by proinflammatory plasmacytoid DCs even in patients on cART. ${ }^{10}$ These overall shifts in immunologic cell populations disrupt normal GI function and the ability of the tissue to respond to pathogens, clear cellular debris, and control inflammation.

Because studying the nature of HIV-associated GI disease in patients is experimentally challenging, nonhuman primate (NHP) models of HIV using SIV provide a unique window of insight into the course of this disease. Exhibiting a similar course of disease both virologically and immunologically, many of these models have been reported to have pathologic changes in the gut and significant alterations in the gutassociated lymphoid tissue that are similar to humans with HIV. ${ }^{11,12}$

In both HIV-infected humans and SIV-infected NHPs the intestinal disease caused by immunologic dysfunction is thought to result in disruption of the gut barrier. This disruption may be sufficient to allow translocation of microbial products and microbes themselves into the lymphatic system and blood stream. This translocation, which is thought to be a main source of systemic inflammation and ultimately an important component of terminal opportunistic infection, is not wholly restored with cART treatment in either humans or NHPs. ${ }^{13-15}$

In addition, recent advances in the study of the microbiota in human health and disease have expanded our understanding of the role of the gut during disease progression. Shifts in the microbiome been associated with disease outcome in humans and have provided insights into potential mechanisms associated with cART and even protection conferred from experimental vaccine approaches. ${ }^{16,17}$

Although enteropathy has been identified and evaluated in HIV-infected humans and some NHP models of HIV infection, it has not been examined in the accelerated SIV/ macaque dual inoculation model of HIV. ${ }^{18}$ Briefly, this model exhibits a course of disease similar to SIV-infected rhesus macaques but in a compressed time span, resulting in death around 3 months postinoculation (p.i.), and infected animals can achieve viral suppression on cART. This model has previously provided unique insights into various aspects of the disease that develop longitudinally in the host, including HIV-associated neurocognitive disease. ${ }^{19}$ The aim of the present study was to evaluate the presence and severity of intestinal disease and its association with viral infection and/or alterations to cell populations in the GI tract of SIV dual virus-inoculated macaques. Specifically, changes to peripheral and GI viral burden, blood $\mathrm{CD}^{+}{ }^{+} \mathrm{T}$-cell count, GI apoptosis, GI $\mathrm{T}$ cells and macrophages, intestinal tight junction continuity, indicators of microbial translocation, and translocation-associated inflammation were evaluated.

\section{Materials and Methods}

\section{Animal Studies}

Tissues from multiple cohorts of juvenile pigtailed macaques were studied retrospectively. All infected groups were inoculated intravenously with two strains of SIV, the immunosuppressive swarm SIV/DeltaB670 and the neurovirulent clone SIV/17E-Fr, as previously described. ${ }^{17}$ Infected, untreated animals were euthanized during various stages of disease $(7,10,14,21,35,42,56$, and approximately 84 days p.i.). Animals that were euthanized at 84 days or before that time if criteria for euthanasia were reached are herein referred to as late-stage infection (LSI).

Animals referred to as cART received a regimen that included the nucleotide reverse-transcriptase inhibitor tenofovir (Gilead Sciences, Foster City, CA) at a dose of $30 \mathrm{mg} / \mathrm{kg}$ subcutaneously once a day from days 12 to 26 p.i. and $10 \mathrm{mg} / \mathrm{kg}$ subcutaneously once a day thereafter; the protease inhibitors saquinavir (Hoffmann La Roche, Basal, Switzerland) and atazanavir (Bristol-Myers Squibb, New York, NY) at doses of 205 and $270 \mathrm{mg} / \mathrm{kg}$ orally twice a day, respectively; and the integrase inhibitor L-870812 (Merck, Kenilworth, NJ) at a dose of $10 \mathrm{mg} / \mathrm{kg}$ orally twice a day beginning at 12 days after virus inoculation. cART animals were monitored for virus in plasma and cerebral spinal fluid by real-time quantitative PCR (qPCR) and experienced marked declines in plasma and cerebral spinal fluid viral RNA levels, reaching $<100$ copy equivalents/mL by approximately 50 days after treatment initiation. cART animals were euthanized between 161 and 175 days postinfection and have been described in further detail previously. ${ }^{20}$ Animals referred to as procedural control (PC) were mock-infected and had blood sampled on the same experimental schedule as cART untreated, SIV-infected animals at LSI. PC animals were euthanized at 84 days p.i. Animal groups evaluated in this study are summarized in Table 1 .

All animals were euthanized in accordance with federal guidelines and institutional policies. At euthanasia animals were anesthetized with ketamine- $\mathrm{HCl}$, followed by

Table 1 Animal Euthanasia Dates and Treatments

\begin{tabular}{llllll}
\hline Group name & Abbreviation & $n$ & Infected & Days PI & Treatment \\
\hline $\begin{array}{l}\text { Procedural } \\
\text { control }\end{array}$ & PC & 12 & No & $84-90$ & No \\
Day 4 & 4d & 8 Yes & 4 & \\
Day 7 & 7d & 6 & 7 & \\
Day 10 & 10d & 6 & 10 & \\
Day 14 & 14d & 6 & 14 & \\
Day 21 & 21d & 6 & 21 & \\
Day 42 & 42d & 9 & 42 & \\
Day 56 & 56d & 9 & 56 & \\
Late stage & LSI & 25 & $60-101$ & \\
$\quad$ infected & & & (terminal) & \\
cART & cART & 5 & $161-175$ & cART \\
\hline
\end{tabular}

cART, combination antiretroviral therapy; LSI, late-stage infection; PI, postinoculation. 
induction of deep anesthesia with pentobarbital and perfused with sterile phosphate-buffered saline. Tissues were collected and either flash frozen or fixed with Streck tissue fixative for subsequent use. All animal studies were approved by the Johns Hopkins Animal Care and Use Committee; all animals were humanely treated in accordance with federal guidelines and institutional policies.

\section{Histologic Scoring for Intestinal Disease}

After necropsy, tissues were fixed using Streck tissue fixative for 1 week at room temperature and then embedded in paraffin. Sections of the stomach, duodenum, jejunum, ileum, cecum, and colon were prepared and stained with hematoxylin and eosin. A semiquantitative scoring template was established in consultation with a board-certified veterinary pathologist to evaluate several visual indicators of intestinal disease. The presence of mixed mononuclear cellular infiltrates, multinucleated giant cells, and parasites were qualitatively assigned a score of 0 (none), 1 (mild), 2 (moderate), or 3 (severe). Thickness and integrity of the epithelium was examined and assigned a score of 0 (intact), 2 (possibly compromised), or 4 (markedly compromised). Villus and glandular fusion, loss, abscesses, dilation, lymphoid follicles, and submucosal mononuclear cells were individually assigned a score of 0 (absent) or 1 (present). The sum of these scores was used as a measure of severity of intestinal disease in each section. The sum of all of the scores for each section was used to assign a severity score for each animal.

To quantitate severity of intestinal disease, sections were blinded (J.D.C.) and systematically examined under light microscopy by a single investigator (M.C.Z.). A separate pathologist evaluated and scored all sections from 24 animals to control for observer bias. Each scoring criterion was individually correlated with total scores to ensure one criterion was not influencing overall results. No PC or cART animal had an intestinal disease score above the median score for LSI animals (median score $=27$ ). As a result, this number was used to define animals with and without intestinal disease. Animals with scores between the 50th and 75th percentile (scores ranging from 27-32) were said to have mild intestinal disease, animals with scores above the 75 th percentile (scores $\geq 33$ ) were said to have severe intestinal disease.

\section{Immunohistochemistry and in Situ Hybridization}

Double labeling of tissues using IHC and ISH was performed by hand on fixed, paraffin-embedded tissue sections. Sections were deparaffinized by baking at $60^{\circ} \mathrm{C}$, then rehydrated through a graded alcohol series. Slides were then pretreated with $25 \mu \mathrm{g} / \mathrm{mL}$ proteinase $\mathrm{K}$ (Roche Applied Science, Indianapolis, IN). ISH for SIV was performed using an antisense SIVmac239 digoxigenin-UTP-labeled riboprobe at a concentration of $1.75 \mathrm{ng} / \mu \mathrm{L}$ overnight at $51^{\circ} \mathrm{C}$. After washing, anti-digoxigenin antibody was applied for 1 hour at $37^{\circ} \mathrm{C}$, followed by the addition of color substrate solution to slides overnight at $4^{\circ} \mathrm{C}$. Uninfected tissues were used as controls. After washing, slides were then blocked with $1 \times$ Power Block (BioGenex, Fremont, CA) for 10 minutes before proceeding to IHC.

Secondary labeling for macrophages was accomplished by using a monoclonal antibody against CD68 [KP1; Agilent (Dako), Santa Clara, CA] at a concentration of $74 \mu \mathrm{g} / \mathrm{L}$ for 60 minutes at room temperature. Secondary labeling for $\mathrm{T}$ cells was accomplished by using a polyclonal antibody against CD3 (A0452; Dako) at a concentration of $60 \mu \mathrm{g} / \mathrm{mL}$ for 60 minutes at room temperature. After washing, biotinylated secondary antibody was added for 30 minutes after which time slides were washed and visualized using the Vector red alkaline phosphatase substrate kit (Vector Laboratories, Burlingame, CA). After staining, slides were washed, dehydrated, and covered with a coverslip.

Apoptosis was measured on tissue in the absence of ISH using a rabbit polyclonal antibody against caspase-3 (9661; Cell Signaling Technology Inc., Danvers, MA) at a concentration of $0.252 \mu \mathrm{g} / \mathrm{mL}$ for 15 minutes at room temperature. All caspase-3 staining was conducted on the Leica (Wetzlar, Germany) Bond Rx automated tissue staining system using the Leica Bond Polymer Refine staining kit with a 20-minute sodium citrate pretreatment.

Epithelial tight junction protein claudin-3 was measured on tissue in the absence of ISH using a rabbit polyclonal antibody against claudin-3 (RB-9251; Thermo Fisher Scientific, Waltham, MA) at a concentration of $4 \mu \mathrm{g} / \mathrm{mL}$ for 15 minutes at room temperate. All claudin-3 staining was conducted on the Leica Bond Rx automated tissue staining system using the Leica Bond Polymer Refine staining kit with a 20-minute sodium citrate pretreatment.

Cytotoxic T lymphocytes (CTLs) were measured in tissue in the absence of ISH using a mouse monoclonal antibody against T-cell intracytoplasmic antigen 1 (TIA-1) antibody at a concentration of $10 \mu \mathrm{g} / \mathrm{mL}$ for 15 minutes at room temperature. All TIA-1 staining was conducted on the Leica Bond Rx automated tissue staining system using the Leica Bond Polymer Refine Red staining kit with a 30-minute sodium citrate pretreatment.

All tissue sections were evaluated and quantified using a Nikon Eclipse microscope fitted with a color Nikon DS-Ri1 camera and NIS Elements Acquisition and Analysis software version 3.22.00 (Nikon, Melville, NY). Slides were blinded (J.D.C., M.C.Z.), and $4 \times 4$ to $8 \times 8$ grids of adjacent microscope fields were imaged at $\times 200$ power, resulting in a composite image containing at least 16 microscopic fields. Composite images were then white balanced and background corrected. Quantitation for CD3, CD8, and caspase- 3 was accomplished by defining regions of interest (ROIs) manually between the muscularis mucosae and the gut lumen, including the epithelium. Peyer's patches and lymphoid follicles were excluded due to their variability in numbers and location in the ileum and colon. ROIs were limited to those in which the crypts/glands 
were in the same plane as the histologic section. Several ROIs were defined, resulting in a total of approximately $2 \mathrm{~mm}^{2}$ of mucosa analyzed. ROIs were analyzed for immunohistochemically positive cells using the same object count settings for each marker between all blinded (J.D.C., M.C.Z.) samples, resulting in both a percentage of ROI area and number of positive cells. The same ROIs on each slide were then manually scored for SIV-ISH-positive cells, yielding a number of positive cells per ROI and a ratio of singly ISH-positive cells to those cells double positive for ISH and IHC.

Percentage of loss of claudin-3 staining was calculated in an approach similar to that detailed by Estes et al. ${ }^{14}$ Briefly, the luminal surface of the epithelium was traced and measured on composite images of the gut lamina propria, resulting in a boundary at least $1 \mathrm{~mm}$ in length. Then all stretches of the epithelium lacking claudin-3 staining were traced and measured. The sum of the lengths of epithelium lacking claudin-3 staining was divided by the overall length of the epithelium and multiplied by 100 to yield a percentage of loss of claudin-3.

The number of cells positive for TIA- 1 was evaluated by counting the total number of TIA-1-positive cells in three microscope fields where the crypts/glands were in the same plane as the histologic section at $\times 400$ magnification. The mean of these three counts was then calculated, resulting in an average TIA-1-positive cells number per field.

\section{RNA Extraction and qPCR of SIV in Plasma}

Viral RNA was isolated from $400 \mathrm{~mL}$ of plasma collected terminally using the QIAamp MiniElute Virus Spin kit (Qiagen, Hilden, Germany). Samples were eluted in $40 \mu \mathrm{L}$ of Tris and ethylenediaminetetraacetic acid buffer and analyzed in triplicate by qPCR, as described elsewhere. ${ }^{19}$ The limit of detection for the assay was established as 10 copies/reaction (100 copy equivalents/mL).

\section{$\mathrm{CD}^{+}{ }^{\mathrm{T}}$-Cell Count}

Terminal blood CD4 T-cell count was obtained by evaluating total number of lymphocytes per millimeter of blood via complete blood count. Then using multicolor flow cytometry analysis, an absolute percentage of lymphocytes positive for CD4 was determined. This percentage was then multiplied by the absolute number of lymphocytes per millimeter of blood to determine the number of CD4 cells per millimeter of blood. This method has been described in detail elsewhere. $^{21}$

\section{DNA Extraction and 16s rDNA qPCR in Plasma and} Tissues

DNA was extracted from $200 \mu \mathrm{L}$ of previously thawed plasma using the QIAmp DNA Mini kit (51304; Qiagen). DNA from liver and mesenteric lymph nodes (MLNs) was extracted from approximately $50 \mathrm{mg}$ of disrupted frozen tissue using the DNeasy kit (69504; Qiagen). Real-time PCR results were obtained using the Microbial DNA qPCR Pan Bacteria 1 assay (Qiagen) on a CFX96 thermocycler (Bio-Rad, Hercules, CA). A 16s rDNA standard was established using DNA from $1 \mathrm{~mL}$ of an overnight Escherichia coli K12 (ER2925; NEB, Ipswich, MA) culture. DNA was extracted from the $E$. coli cell pellet using the QIAmp DNA Mini kit (51304; Qiagen). DNA purity and concentration were measured using a Nanodrop spectrophotometer (ND-1000). After determining the DNA concentration, the number of genome equivalents was calculated using the known E. coli $\mathrm{K} 12$ genome size. A 1:15 eightfold dilution series was then used to establish a standard curve down to $<1$ genome per reaction. Limit of detection was established at $<30$ E. coli $\mathrm{K} 12$ genome equivalents per reaction.

\section{Plasma sCD14, sCD163, and LBP}

Soluble CD (sCD)14 was measured via sandwich enzymelinked immunosorbent assay (ELISA) on $100 \mu \mathrm{L}$ of thawed plasma in 1:200 dilution using the human SCD14 Quantikine ELISA kit (DC140; R\&D Systems, Minneapolis, MN). sCD163 was measured via sandwich ELISA on $50 \mu \mathrm{L}$ of undiluted thawed monkey plasma using the human sCD163 Quantikine ELISA kit (DC1630; R\&D Systems). Lipopolysaccharide binding protein (LBP) was measured in $100 \mu \mathrm{L}$ of undiluted thawed monkey plasma using the human LBP ELISA kit (KA0448; Abnova, Jhongli, Taiwan). All plates were analyzed using an iMark microplate reader (Bio-Rad).

\section{Statistical Analysis}

Comparisons between two treatment groups were done using $U$-tests. Comparisons between multiple treatment groups were done using Kruskal-Wallis tests, followed by Dunn's multiple comparisons tests between the mean ranks of every group. Correlations were determined using Spearman's rank correlation tests or Pearson's correlation tests. $P$ values for multiple correlations were corrected using Benjamini-Hochberg multiple comparisons correction. Excel 2013 (Microsoft, Redman, WA) was used to organize data and to generate descriptive statistics. Prism 6.0 (GraphPad Inc., San Diego, CA) was used to perform all other statistical analyses and to generate graphs. Graphs showing Kruskal-Wallis group comparisons report $P$ values after Dunn's corrections.

\section{Results}

\section{Intestinal Disease Scoring Criteria}

To evaluate the impact of each intestinal disease scoring criteria on the total score, the criteria were broken down into 
the following four main categories: giant cells, cellular infiltrates, epithelium integrity, and villus/gland disruption. The descriptive statistics for LSI and PC animals were compared, and the average scores for each disease indicator category were compared (Table 2). LSI animals scored higher in all categories than PC animals. Multinucleated giant cells were not observed in PC animals but were seen in $58.3 \%$ of LSI animals. Mixed cellular infiltrates were observed in all animals because some density of lymphocytes and macrophages are normal in the lamina propria of the gut. The epithelium was compromised in $29.1 \%$ more LSI animals than in PC animals. Abnormal villi and glands were only observed in $8.3 \%$ of PC animals but were observed in $66.7 \%$ of LSI animals.

Pearson's correlation tests were performed between each of the four categories (giant cells, cellular infiltrates, epithelium integrity, and villus/glad disruption) and the overall intestinal disease score for LSI animals. All four categories significantly correlated with the overall intestinal disease score. However, none of the correlations was especially strong. Of the categories, villus and glandular abnormalities were the best predictors of total intestinal disease severity $\left(r=0.50, r^{2}=0.25, P<0.0001\right)$. Cellular infiltrates were the worst predictors of total intestinal disease severity $\left(r=0.26, r^{2}=0.07, P<0.0001\right)$. Detailed evaluation of the intestinal severity scoring criteria both total and by anatomic location are described in Supplemental Tables S1-S3.

\section{Intestinal Disease Scores}

The first goal was to unbiasedly characterize the nature of any observable histopathologic changes in several cohorts of SIV-infected macaques. These groups included uninfected PC animals, infected animals euthanized at various stages of infection, and infected animals treated with cART starting at day 12 p.i. Information on animal groups is summarized in Table 1.

Tissues from each animal were evaluated for indicators of intestinal disease using a scoring system detailed in Materials and Methods. Briefly, changes such as mixed cellular infiltration, villus and glandular disruption, presence and overgrowth of microflora, and disruption of the epithelium were evaluated and individually assigned scores; the sum of which indicated that GI region's severity of intestinal disease. The sum of the scores for all regions of the
GI tract represented each animal's overall intestinal disease score. Representative images of normal animals and animals with several of these disease indicators are depicted in Figure 1, A-F.

There was a significant difference in the mean rank between all of the animal groups examined $(\mathrm{H}=22.73$, $P<0.0001$ ) (Figure 1G). Specifically, LSI animals exhibited significantly more intestinal disease than matched PC animals $(P=0.0002)$ (Figure $1 \mathrm{H})$. Interestingly, animals that received cART had levels of intestinal disease that were significantly lower than LSI animals and were similar to PC animals $(P=0.0129)$ (Figure $1 \mathrm{H})$. Of the other time points examined, only two animals euthanized at day 56 postinfection had scores $>27$; both of these had mild enteropathy (Figure 1G).

\section{Relation between Intestinal Disease, $\mathrm{pVL}$, and Blood $\mathrm{CD}^{+}{ }^{+}$T-Cell Count}

Severity of intestinal disease was compared with terminal plasma viral load (pVL) and terminal blood $\mathrm{CD}^{+}{ }^{+} \mathrm{T}$-cell count for all LSI animals, both with and without enteropathy. There was no overall correlation with all LSI animals. However, when animals with mild or severe intestinal disease were examined separately, there was a highly significant positive correlation between $\mathrm{pVL}$ and the severity of intestinal disease $(r=0.841, P=0.0006)$ (Figure 1I). In addition, in animals with severe intestinal disease there was a significant negative correlation between terminal blood CD4 count and severity of intestinal disease $(r=-0.9710$, $P=0.011$ ) (Figure $1 \mathrm{~J}$ ).

\section{Intestinal Disease by Intestinal Region}

In every intestinal site examined animals with the most severe intestinal disease were LSI animals. With the exception of the duodenum, all intestinal sites examined trended toward or had significantly different mean ranks of intestinal disease between PC, cART, and LSI animals (Figure 2). The strongest statistical differences in mean rank of intestinal disease were found in the ileum $(\mathrm{H}=16.51$, $P=0.0003)$ and colon $(\mathrm{H}=23.00, P<0.0001)$ (Figure 2). All group differences represented a significant increase or trend toward an increase in the mean rank of intestinal disease in LSI animals. The most significant elevations in mean rank of intestinal disease between PC and

Table 2 Intestinal Disease Scoring Descriptive Statistics

\begin{tabular}{|c|c|c|c|c|c|c|c|c|c|}
\hline \multirow[b]{2}{*}{ Disease indicator } & \multicolumn{3}{|l|}{$\mathrm{PC}$} & \multicolumn{3}{|l|}{ CART } & \multicolumn{3}{|l|}{ LSI } \\
\hline & Mean & Median & Pos & Mean & Median & Pos & Mean & Median & Pos \\
\hline Giant cells & 0 & 0 & 0 & 0 & 0 & 0 & 4.08 & 2 & 58.3 \\
\hline Epithelium integrity & 0.83 & 0 & 16.7 & 0.4 & 0 & 20 & 1.29 & 0 & 45.8 \\
\hline Villus/gland abnormality & 0.08 & 0 & 8.3 & 0 & 0 & 0 & 2.83 & 2 & 66.7 \\
\hline
\end{tabular}

CART, combination antiretroviral therapy; LSI, late-stage infection; PC, procedural control; Pos, percent positive. 
A
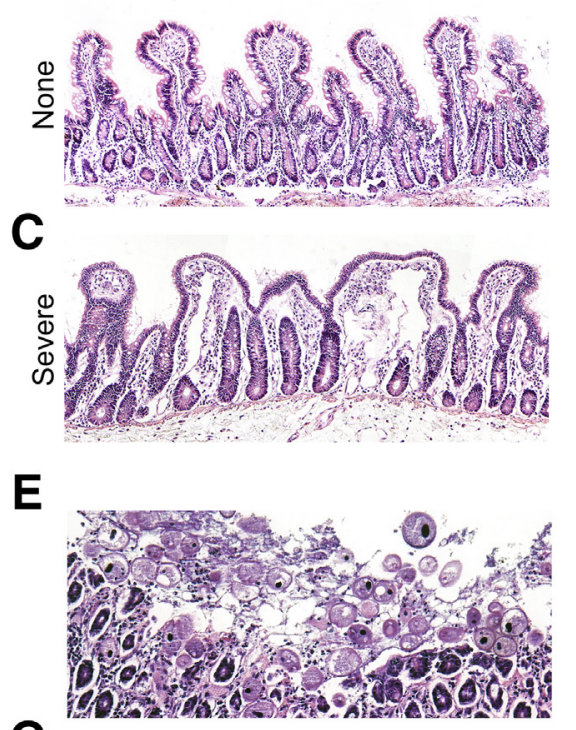

G

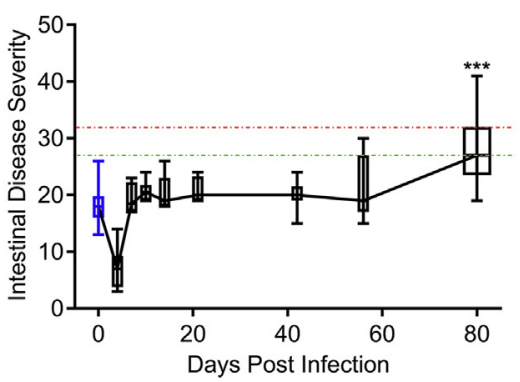

I

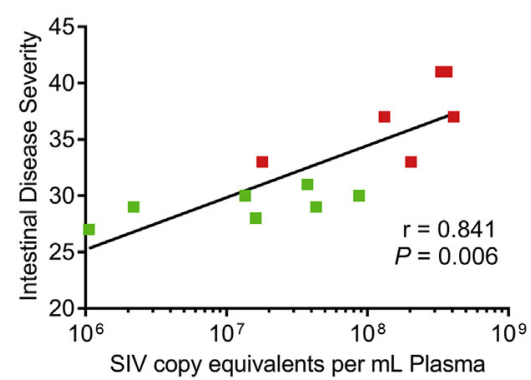

B

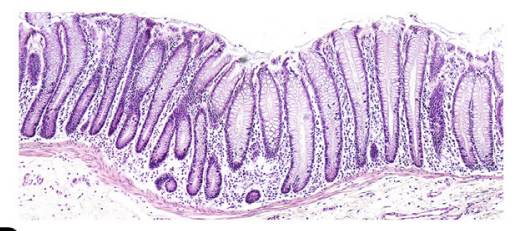

D

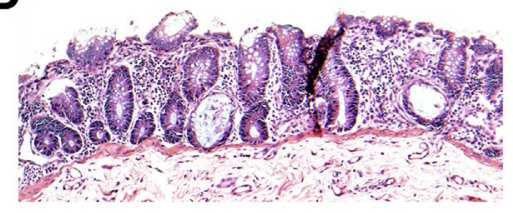

$\mathbf{F}$

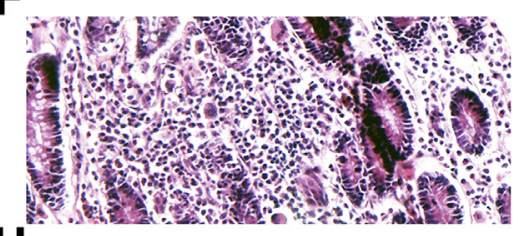

$\mathbf{H}$

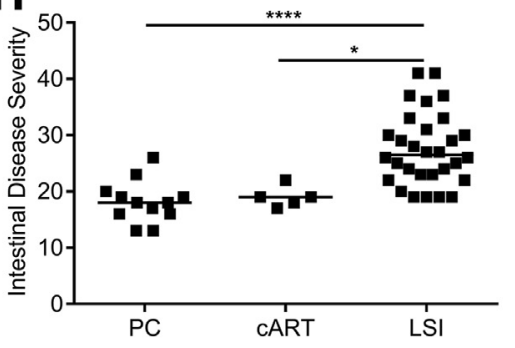

$J$

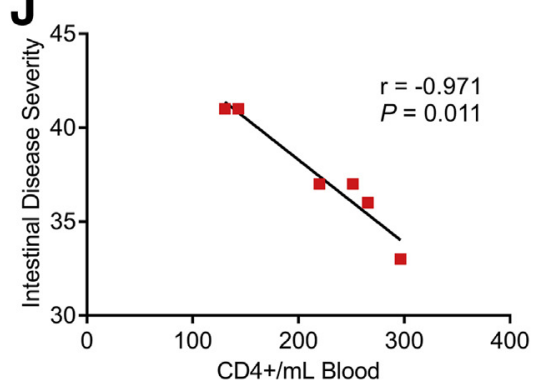

Figure 1 Intestinal disease scores in uninfected, SIV-infected CART-treated, and SIVinfected untreated pigtailed macaques. Intestinal disease was evaluated in PC, SIV-infected animals euthanized at days $4,7,10,14,21,42$, and 56 postinfection; SIV-infected animals at late stage infection (LSI); and SIV-infected animals on CART. A and B: Hematoxylin and eosin stained tissue sections of ileum (left) and colon (right) from a LSI animal with no intestinal disease. C and D: Hematoxylin and eosin stained tissue sections of ileum (left) and colon (right) from a LSI animal with severe intestinal disease. E: Representative Balantidium coli overgrowth and associated tissue damage in colon of a LSI-Severe animal. F: Representative multinucleated giant cells in the colon of a LSI-Severe animal. G: PC (blue) and longitudinal total intestinal disease scores. Green and red dotted lines indicate the intestinal disease score for animals with mild or severe intestinal disease. H: Comparison of intestinal disease scores between PC, CART, and LSI animals. I: Spearman correlation between intestinal disease score and pVL. J: Spearman correlation between intestinal disease score and terminal CD4 T-cell count. Green and red squares indicate LSI animals with mild or severe intestinal disease, respectively. Lines at the center of grouped data represent the group median. Exact $P$ values between groups were calculated by Kruskal-Wallis test, followed by Dunn post hoc correction. ${ }^{*} P<0.05,{ }^{* *} P<0.001$, and $* * * * P<0.0001$. Original magnification, $\times 100(\mathbf{A}-\mathbf{F})$. CART, combination antiretroviral therapy; $\mathrm{PC}$, procedural control; $\mathrm{pVL}$, plasma viral load.
LSI animals were in the ileum $(P=0.0025)$ and colon $(P<0.0001)$. With these findings, we selected the ileum and colon for subsequent study.

\section{Caspase-3 Expression in Ileum and Colon}

Caspase-3 expression was measured using IHC to evaluate the levels and locations of apoptosis in the ileum and colon of PC animals compared with LSI animals with or without severe intestinal disease (LSI-Severe or LSI-None). In PC animals, caspase- 3 was concentrated in proximity to the gut lumen, namely the tips of the villi in the ileum and the superficial lamina propria of the colon (Figure 3, A and B). However, in LSI-Severe animals, caspase-3 localized around areas of villus loss in the ileum and glandular degeneration in the colon (Figure 3, C and D). There was a significant difference in the mean rank of percentage of ROIs positive for caspase- 3 between PC, LSI-None, and LSI-Severe animals in the ileum $(\mathrm{H}=8.769, P=0.0012)$ and colon $(\mathrm{H}=5.692, P=0.0487)$ (Figure $4, \mathrm{~A}$ and $\mathrm{B}$ ). The percentage of ROIs positive for caspase- 3 was found to be significantly increased in LSI-Severe animals compared with PC animals in the ileum $(P=0.0098)$ and trended toward an increase between LSI-None and LSI-Severe animals in the colon $(P=0.0930)$.

\section{CD3 Expression and Distribution in the Ileum and Colon}

Slides from the ileum and colon of PC, LSI-None, and LSISevere animals were dually labeled for CD3 and SIV using IHC and ISH. In PC animals, $\mathrm{CD}^{+}$cells were distributed evenly throughout the lamina propria in the ileum and colon 

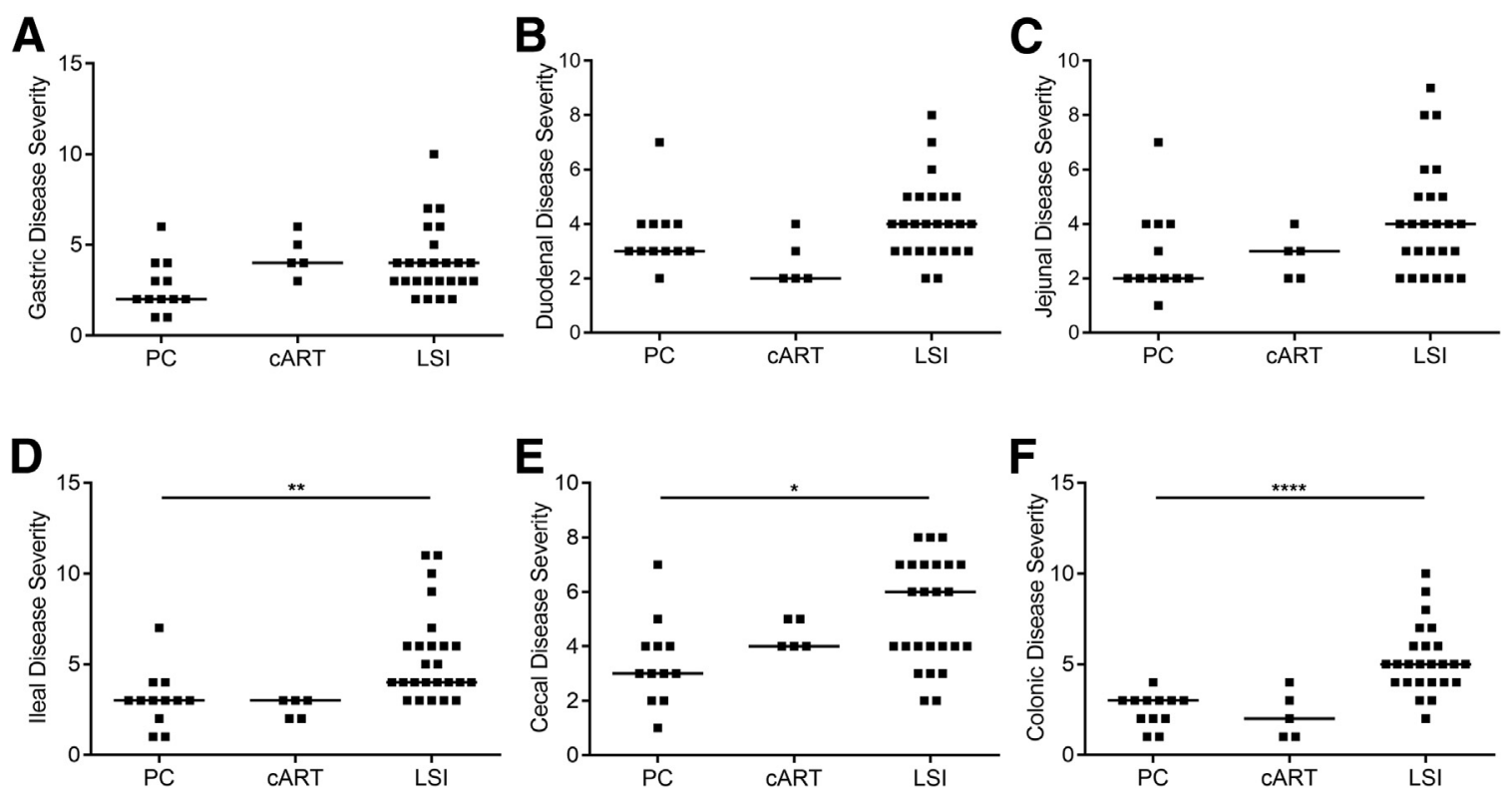

Figure 2 Intestinal disease by tissue site in uninfected, SIV-infected combination antiretroviral therapy (cART)-treated, and SIV-infected untreated pigtailed macaques. Intestinal disease in six intestinal sites was evaluated in PCs, terminally SIV-infected animals, and SIV-infected animals on cART. Stomach (A), duodenum (B), jejunum (C), ileum (D), cecum (E), and colon (F). Exact $P$ values between groups were calculated by Kruskal-Wallis test, followed by Dunn post hoc correction. Lines at the center of grouped data represent the group median. Compared with PC animals, LSI animals trended toward having more intestinal disease in the stomach $(P=0.0512)$ and jejunum $(P=0.0811)$. ${ }^{*} P<0.05,{ }^{*} P<0.01$, and ${ }^{* * * * P}<0.0001$. LSI, late-stage infection; $\mathrm{PC}$, procedural control.

(Figure 3, E and F). However, in LSI-Severe animals, what $\mathrm{CD}^{+}$cells remained congregated in foci located around the muscularis mucosae (Figure 3, G and $\mathrm{H}$ ). There was a significant difference in the mean rank of the percentage of ROIs positive for CD3 between PC, LSI-None, and LSISevere animals in the ileum $(\mathrm{H}=7.269, P=0.0152)$ and colon $(\mathrm{H}=7.538, P=0.0107)$ (Figure $4, \mathrm{C}$ and $\mathrm{D}$ ). These group differences represented a significant decrease between the mean rank of the percentage of ROIs positive for CD3 between LSI-None and LSI-Severe animals in the ileum $(P=0.0243)$ and colon $(P=0.0324)$. In addition, although only approaching significance, CD3 percentage of ROIs tended to be significantly lower between PC and LSISevere animals in the colon $(P=0.0930)$.

\section{CD68 Expression and Distribution in Ileum and Colon}

Slides from the ileum and colon of PC, LSI-None, and LSISevere animals were also dually labeled for CD68 and SIV using IHC and ISH, respectively. In PC animals, CD68 ${ }^{+}$ cells were concentrated in proximity to the gut lumen, namely, the tips of the villi in the ileum and near the basal surface of the colon epithelium (Figure 3, I and J). However, in LSI-Severe animals, $\mathrm{CD}^{+} 8^{+}$cells were evenly distributed throughout the lamina propria in the ileum and were concentrated around degenerating glands in the colon (Figure 3, K and L). In the colon there was a significant difference in mean rank of the percentage of ROIs positive for CD68 between PC, LSI-None, and LSI-Severe animals $(\mathrm{H}=5.808, P=0.0442$ ) (Figure $4, \mathrm{E}$ and $\mathrm{F}$ ). This represented a significant reduction in CD68 between PC animals and LSI-Severe animals $(P=0.0482)$. No differences in mean rank of the percentage of ROIs positive for CD68 in the ileum were found.

\section{SIV Hybridization and Double-Labeled Cells in Ileum and Colon during LSI}

Slides double-labeled for CD3/SIV or CD68/SIV were scored for the number of cells that were positive for SIV RNA alone or double-labeled for SIV and CD68. Overall, the number of SIV-positive cells was significantly higher in the ileum but not in the colon of LSI-None animals compared with LSI-Severe animals $(P=0.0286)$ (Figure 5 , A and B). The total number of CD3/SIV or CD68/SIV double-positive cells was not significantly different in the ileum or colon of LSI-None and LSI-Severe animals. However, CD68/SIV represented a larger proportion of double-labeled cells than CD3/SIV in LSI-Severe animals (Figure 5, C and D).

Correlations between Staining for Caspase-3, CD3, and CD68 in Ileum and Colon and Intestinal SIV or pVL

In all LSI animals the percentage of ROIs positive for CD3 but not CD68 was strongly correlated with SIV infection. After Bonferroni correction, the percentage of ROIs positive for CD3 was negatively correlated with the number of cells positive for SIV in the ileum $(r=-0.8330, P=0.0154)$ and showed a weak negative correlation in the colon $(r=-0.6900, P=0.1249$ ) (Figure 6, A and B). There also 

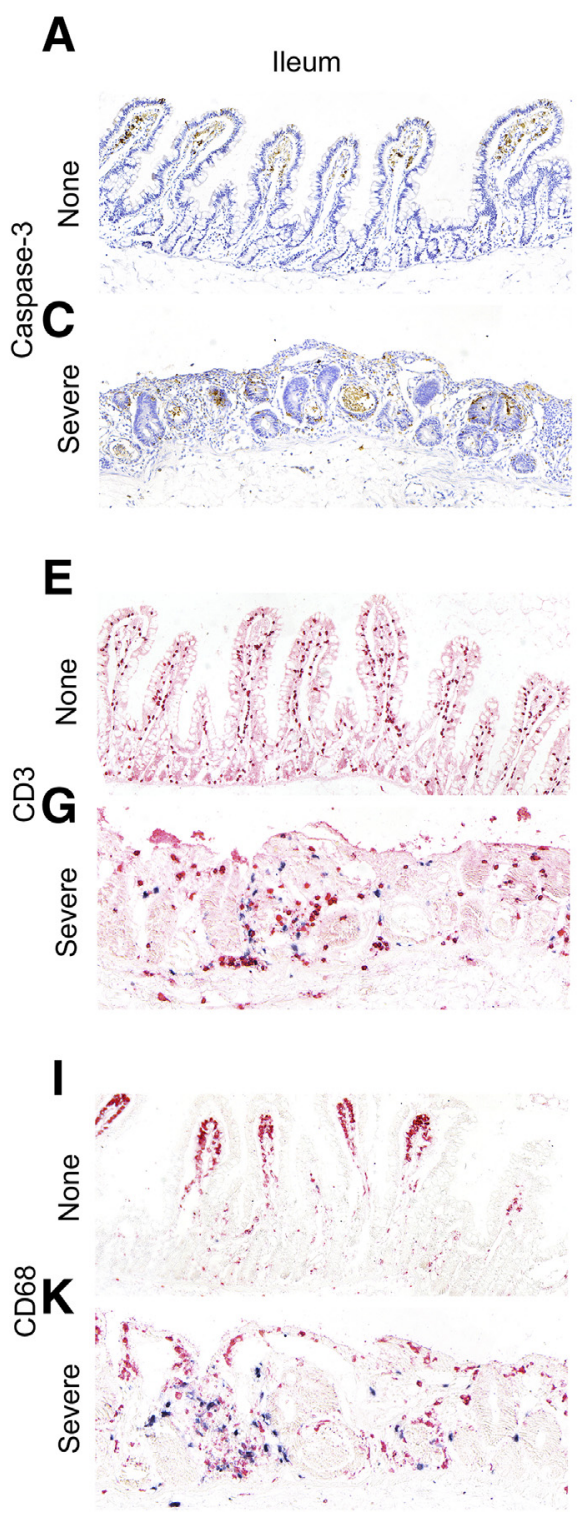

$\mathbf{F}$
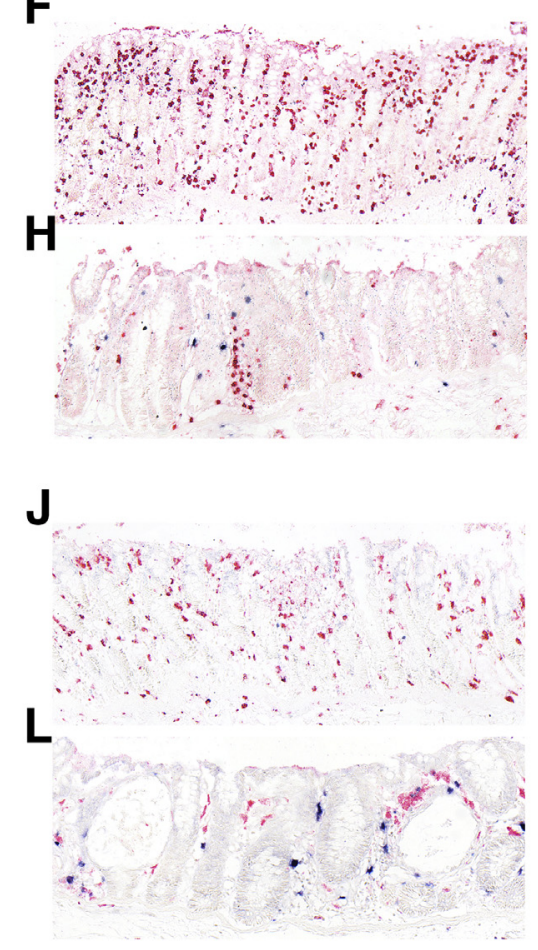

Figure 3 Representative images of caspase-3, CD3, CD68, and SIV expression. Immunohistochemistry and in situ hybridization in the ileum and colon of late-stage infected (LSI) animals. A and B: Caspase-3 staining of LSI animals without intestinal disease. $\mathbf{C}$ and $\mathbf{D}$ : Caspase-3 staining of LSI animals with severe intestinal disease. $\mathbf{E}$ and $\mathbf{F}$ : CD3 and SIV staining of LSI animals without intestinal disease. $\mathbf{G}$ and $\mathbf{H}$ : CD3 and SIV staining of LSI animals with intestinal disease. I and J: CD68 and SIV staining of LSI animals without intestinal disease. $\mathbf{K}$ and L: CD68 and SIV staining of LSI animals with intestinal disease. Original magnification, $\times 100$. was a negative correlation in all LSI animals between the percentage of ROIs positive for CD3 and pVL both in the ileum $(r=-0.9430, P=0.0177)$ and colon ( $r=-0.8860, P=0.0428$ ) (Figure 6, C and D). There were weak negative correlations between the percentage of ROIs positive for CD3 and the percentage of ROIs positive for caspase-3 in ileum $(r=-0.619, P=0.2300)$ and colon ( $r=-0.690, P=0.1062$ ) (Figure 6, E and F).

In the ileum, the percentage of ROIs positive for caspase-3 was positively correlated with pVL $(r=0.786$, $P=0.0335)$. In the colon, the percentage of ROIs positive for caspase-3 was positively correlated with the number of cells positive for SIV $(r=0.886, P=0.0461$ ) (Figure 6, $\mathrm{G}$ and $\mathrm{H})$. Further, in all LSI animals regardless of intestinal disease severity, the number of cells positive for SIV RNA was positively correlated with pVL in the ileum ( $r=0.9430, P=0.0188$ ) and trended positively in the colon $(r=0.8290 P=0.0875)$ (Figure 6I).
Direct and Indirect Measures of Microbial Translocation in LSI

Changes in sCD163, which measures increased macrophage activation that may be partially due to increased microbial translocation, were measured via ELISA. The mean rank of sCD163 was significantly different between PC, cART, and LSI animals $(\mathrm{H}=13.09, P=0.0014)$. This difference indicated a strong trend toward elevation in SCD163 mean rank in LSI animals with or without intestinal disease versus $\mathrm{PC}$ animals $(P=0.0527)$ (Figure $7 \mathrm{~A})$.

Elevations in sCD14 and LBP are thought to result from translocation of microbes and their products, specifically lipopolysaccharide. Plasma SCD14 and LBP were evaluated using ELISA. The mean rank of sCD14 was significantly different between PC, cART, and LSI animals $(\mathrm{H}=6.691$, $P=0.0352$ ). These differences indicated significant elevation of sCD14 in cART animals $(P=0.0388)$ and a 
A

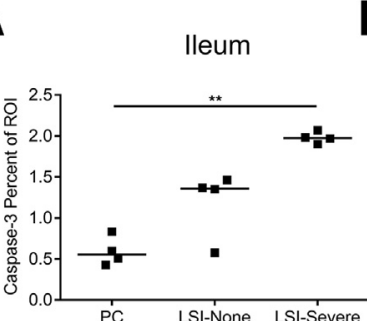

Colon

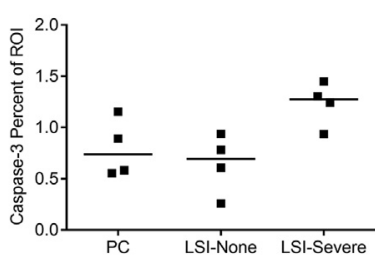

C

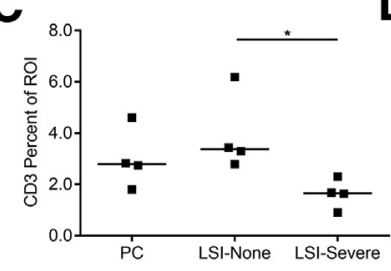

D

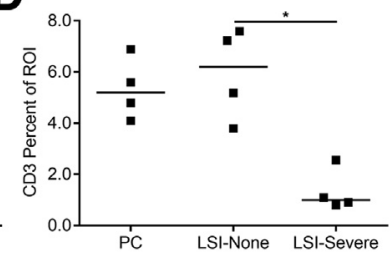

E

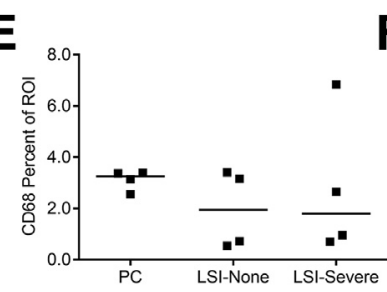

$\mathbf{F}$

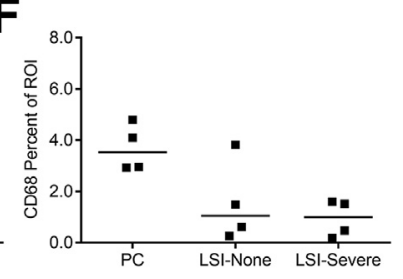

Figure 4 Quantitation of caspase-3, CD3, and CD68 immunohistochemistry. Region of interest (ROI) positive by immunohistochemistry in the ileum and colon of procedural control (PC) and SIV-infected animals at late-stage infection (LSI) with and without severe intestinal disease. $\mathbf{A}$ and B: Caspase-3 staining in PC and LSI animals with or without intestinal disease. C and D: CD3 and SIV staining in PC and LSI animals with or without intestinal disease. E and F: CD68 and SIV staining in PC and LSI animals with or without intestinal disease. Lines at the center of grouped data represent the group median. Exact $P$ values between groups were calculated by Kruskal-Wallis test, followed by Dunn post hoc correction. Compared with LSI-None animals, LSI-Severe animals trended toward having more caspase-3 staining in the colon $(P=0.0930)$. Compared with PC animals, LSI-Severe animals trended toward having less staining for CD68 in the colon $(P=0.0723) .{ }^{*} P<0.05,{ }^{*} P<0.01$.

trend toward elevation in LSI animals with or without intestinal disease $(P=0.0540)$ (Figure $7 \mathrm{~B})$. LBP mean rank trended similarly to $\mathrm{SCD} 14$ between PC, cART, and LSI animals $(\mathrm{H}=4.940, P=0.0846)$. This was due to a weak increase in LBP in LSI animals with or without intestinal disease $(P=0.1531)$ (Figure $7 \mathrm{C})$ but no change in LBP levels in cART animals.

PCR for microbial 16s rDNA was conducted on plasma, liver, and MLN tissue to directly detect microbial translocation. In the plasma a significant difference was found between PC, cART, and LSI animals $(\mathrm{H}=10.56$, $P=0.0051)$. This was due to a significant increase in cART animals $(P=0.0024)$ but not LSI animals (Figure 7D). For both the liver and MLN, the median levels of 16s rDNA were not significantly different between PC, cART, and LSI animals (Figure 7, E and F).

No indicator of microbial translocation correlated with either $\mathrm{pVL}$ or terminal CD4 count via Spearman correlation. Further, no group differences were found when LSI animals were subdivided into those with or without severe intestinal disease.

Of note, for all of the assays except sCD14 several samples primarily in the PC and cART groups tested below the limits of detection for the given assay. Because we are using a nonparametric approach to these analyses, which rely on rank-based comparisons, a meaningful statistical conclusion could not be drawn for several comparisons, and the data should be therefore considered qualitatively.

\section{CD3 T-Cell and Epithelial Claudin-3 Loss in the Ileum} and Colon during Acute and Asymptomatic Infection

Ileum and colon of PC, acute (7 days p.i.), and asymptomatic (21 days p.i.) animals were labeled for CD3 and claudin-3 using IHC. There was a significant difference in the mean rank of the percentage of ROIs positive for $\mathrm{CD} 3$ between PC, acute, and asymptomatic animals in the ileum $(\mathrm{H}=8.115, P=0.0031)$ and colon $(\mathrm{H}=7.385$, $P=0.0145$ ) (Figure 8, A and B). These group differences represented a significant decrease between the mean rank of the percentage of ROIs positive for CD3 between acute and asymptomatic animals in the ileum $(P=0.0134)$. Although only approaching significance, CD3 percentage of ROIs trended toward being significantly lower between acute and asymptomatic or PC animals in the colon $(P=0.0558$, $P=0.0558)$. There was no significant difference in the
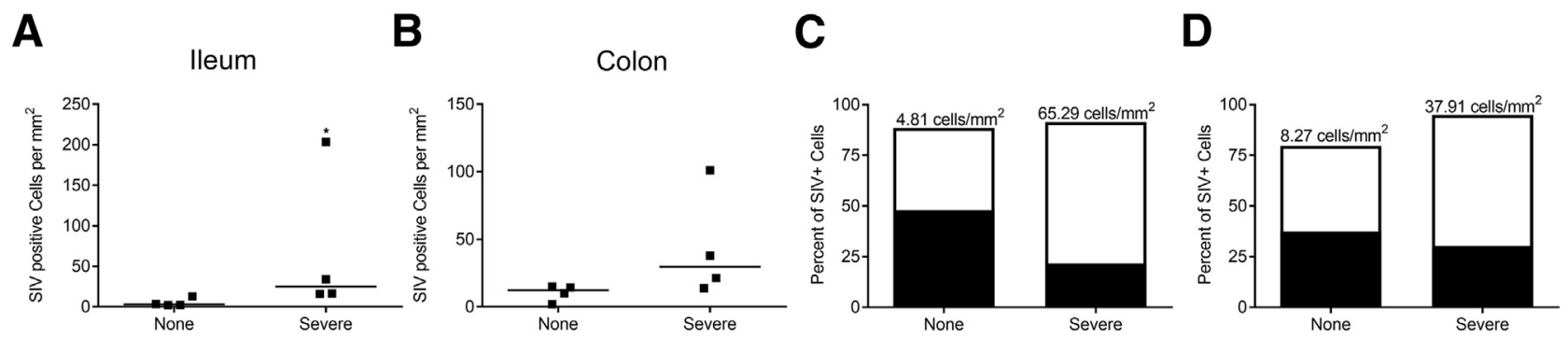

Figure 5 Quantitation of SIV by in situ hybridization. The number of cells positive by in situ hybridization for SIV in the ileum and colon of SIV-infected animals at late-stage infection (LSI) with and without severe intestinal disease. A and B: Total cells singly positive for SIV. C and D: Percentage of cells double labeled for SIV and CD3 or SIV and CD68. Numbers on bar graphs are the average total number of cells double-positive for SIV and CD3 or CD68 normalized to 1 $\mathrm{mm}^{2}$. Lines at the center of grouped data represent the group median. Exact $P$ values between groups were calculated by $U$-test. ${ }^{\star} P<0.05$. 

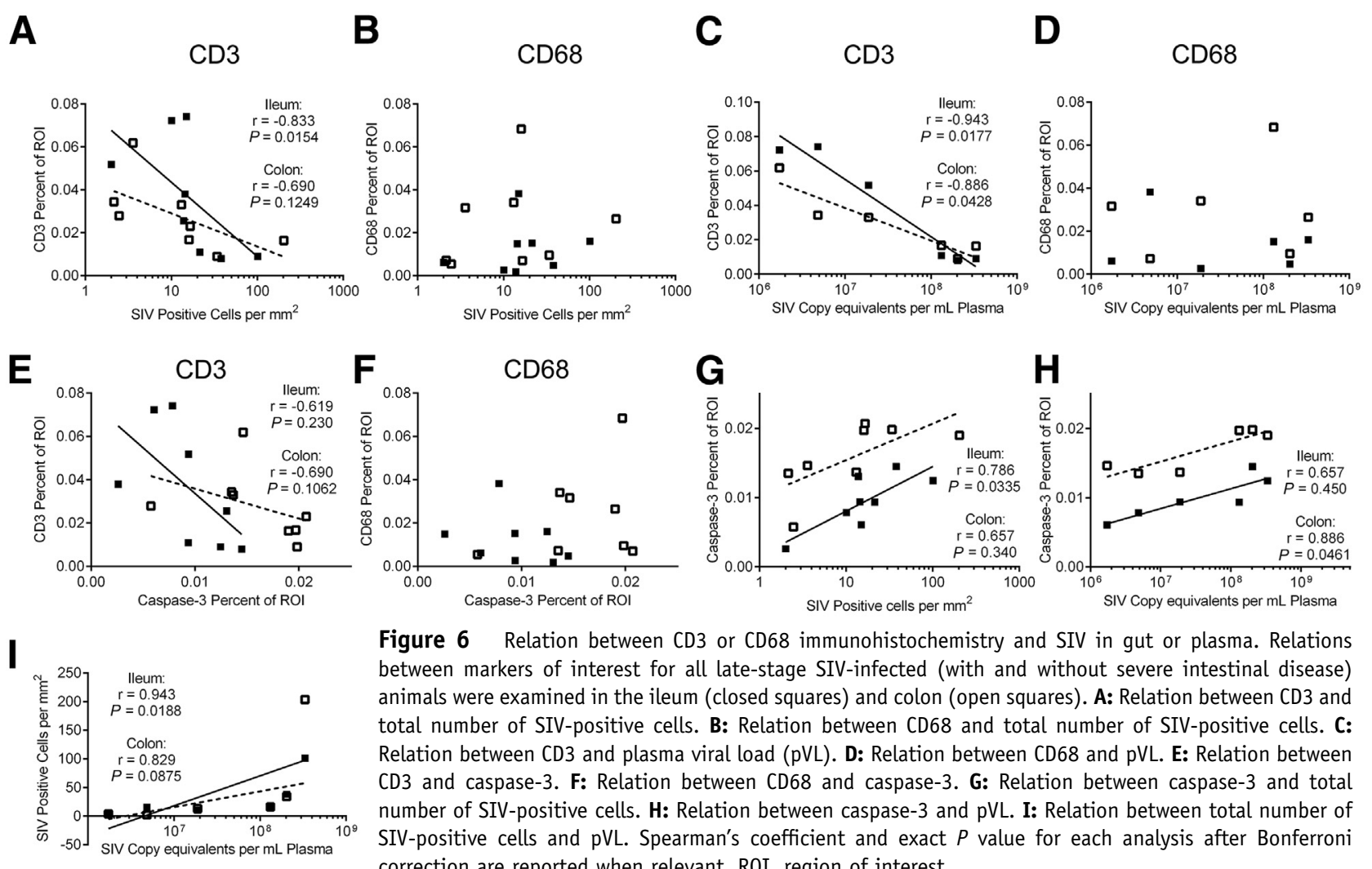

Figure 6 Relation between CD3 or CD68 immunohistochemistry and SIV in gut or plasma. Relations between markers of interest for all late-stage SIV-infected (with and without severe intestinal disease) animals were examined in the ileum (closed squares) and colon (open squares). A: Relation between CD3 and total number of SIV-positive cells. B: Relation between CD68 and total number of SIV-positive cells. C: Relation between CD3 and plasma viral load (pVL). D: Relation between CD68 and pVL. E: Relation between CD3 and caspase-3. F: Relation between CD68 and caspase-3. G: Relation between caspase-3 and total number of SIV-positive cells. H: Relation between caspase-3 and pVL. I: Relation between total number of SIV-positive cells and pVL. Spearman's coefficient and exact $P$ value for each analysis after Bonferroni correction are reported when relevant. ROI, region of interest.

mean rank of percentage of epithelium lacking claudin-3 staining between $\mathrm{PC}$, acute, and asymptomatic animals (Figure 8, C and D).

\section{Evaluation of Epithelial Claudin-3 Loss and TIA-1 Expression in the Ileum and Colon in LSI}

Ileum and colon of PC, LSI-None, and LSI-Severe animals were labeled for claudin-3 and TIA-1 using IHC. There was a significant difference in the mean rank of percentage of epithelium lacking claudin-3 staining between PC, LSINone, and LSI-Severe animals in the ileum $(\mathrm{H}=5.808$, $P=0.0442)$ and colon $(\mathrm{H}=5.808, P=0.0442)$ (Figure 8, E and F). These group differences represented a significant increase in the mean rank of percentage of loss of claudin-3 between PC and LSI-Severe animals in the ileum $(P=0.0482)$ and colon $(P=0.0482)$.

There also was a significant difference in the mean rank of number of TIA-1-positive cells per field between PC, LSI-None, and LSI-Severe animals in the ileum $(\mathrm{H}=7.681, P=0.0071)$ and colon $(\mathrm{H}=8.578$, $P=0.0017$ ) (Figure $8, \mathrm{G}$ and $\mathrm{H}$ ). These group differences represented a significant increase in the mean rank of TIA-1-positive cells per field between PC and LSINone animals in the ileum $(P=0.0119)$ and colon $(P=0.0075)$.

\section{Discussion}

The most remarkable findings of this study are that, although discrete and severe intestinal disease develops in many SIV-infected animals at LSI, those animals that do not develop intestinal disease have intact mucosal T-cell populations similar to those in PC animals, despite significant declines in $\mathrm{T}$ cells in peripheral blood. Further, these T-cell populations seem to be a key factor contributing not only to intestinal viral burden but to levels in the plasma as well. These phenomena appear to be independent of macrophage lineage cell populations in the gut despite a large proportion of virus-infected macrophages. Finally, and perhaps most unexpectedly, the presence or severity of enteropathy apparently had little to no impact on levels of microbial translocation in the animals with SIV infection status better predicting translocation.

To our knowledge, this is one of a few studies to comprehensively examine the entire GI tract for the cellular and virologic effects of SIV in pigtailed macaques. Further, it is the first to describe the effects of SIV on the GI tract in a rapidly progressing macaque model of HIV infection. Of the many microscopic criteria used to evaluate intestinal disease, abnormalities in villous or glandular structure were the most sensitive indicators of enteropathy. Human pathologists have cited similar criteria in HIV-infected patients as reliable indicators of intestinal disease. ${ }^{3,4}$ Anatomically, the ileum and colon were the sites that exhibited the most severe 

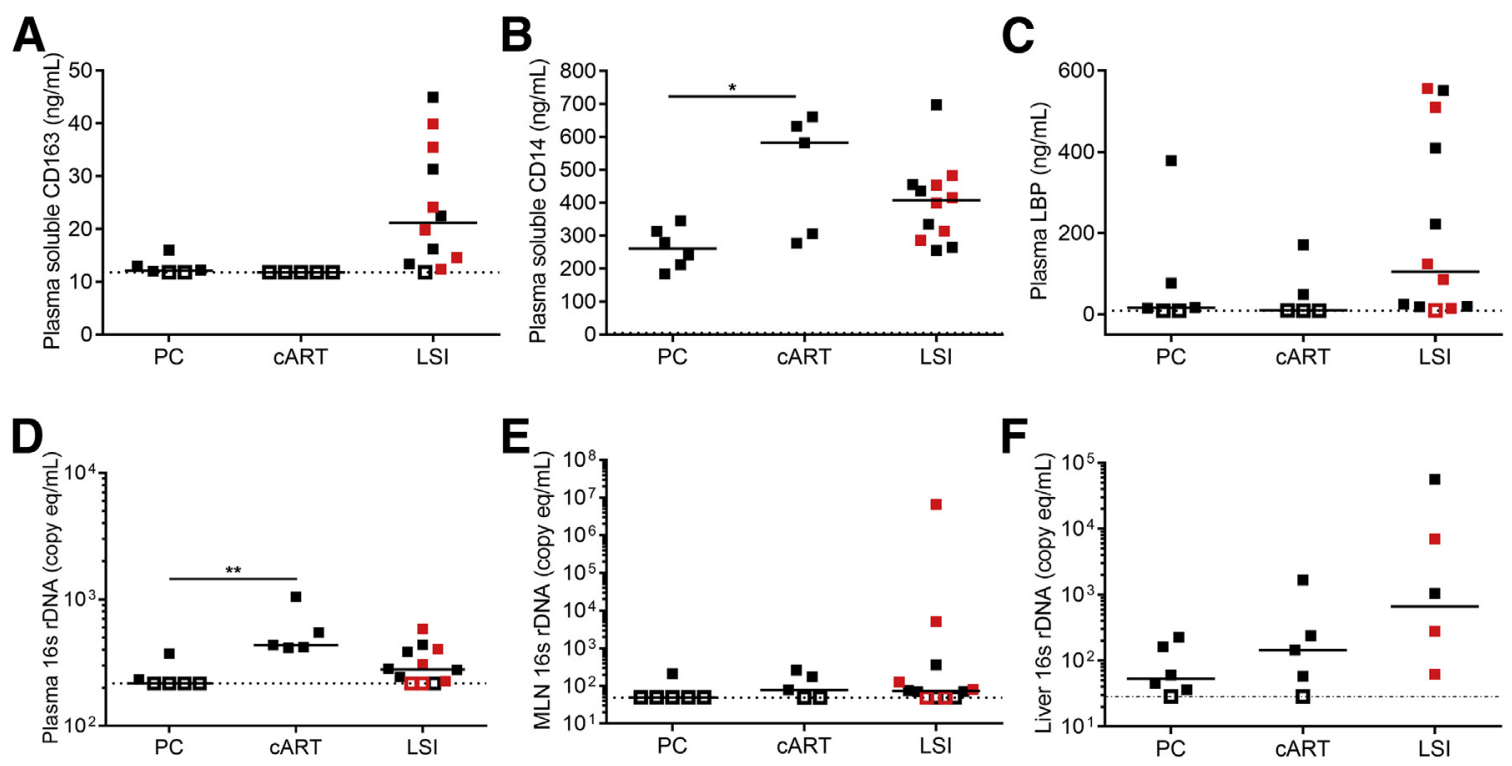

Figure 7 Direct and indirect measures of microbial translocation and associated inflammation during late-stage disease. Enzyme-linked immunosorbent assays (ELISAs) (top row) and quantitative PCRs (bottom row) were conducted on samples from procedural controls (PCs), late-stage infection (LSI) animals with (red squares) and without (black squares) severe intestinal disease and SIV-infected animals on combination antiretroviral therapy (cART). Plasma soluble CD163 (A), plasma soluble CD14 (B), plasma lipopolysaccharide binding protein (LBP) (C), plasma 16s ribosomal DNA (D), mesenteric lymph node (MLN) 16s ribosomal DNA (E), and liver 16s ribosomal DNA $(\mathbf{F})$. Open symbols represent samples that were below the given assays limit of detection. Lines at the center of grouped data represent the group median. Exact $P$ values between groups were calculated by Kruskal-Wallis test, followed by Dunn post hoc correction. Compared with PC animals, LSI animals trended toward having more plasma soluble CD163 $(P=0.0527)$ and $\operatorname{sCD} 14(P=0.0540)$. ${ }^{*} P<0.05, * * P<0.01$.

pathologic changes. This suggests that examination of villus and glandular structure in the ileum and colon are the most efficient method for evaluating SIV-induced enteropathy in pigtailed macaques and likely other NHPs.

Some histopathologic features in the gut of rapidly progressing SIV-infected pigtailed macaques reflect previous observations in SIV/HIV-infected macaques and humans, although others appear unique to this model. In particular, histopathologic evidence of enteropathy did not arise until 56 days p.i., when the macaques are undergoing the early stages of systemic immune depletion and recrudescence of $\mathrm{pVL}$ and tissue viral load. Statistically significant pathologic changes in the gut were not present until late-stage disease, approximately 3 months p.i. This is in contrast to humans and the SIV/rhesus macaque model in which enteropathy appears early in infection and the histologic lesions are sustained. $^{3,12,22}$

We examined three groups of SIV-infected animals at LSI, namely, those with mild (LSI-Mild), severe (LSISevere), or no (LSI-None) intestinal disease. There was a highly significant positive correlation between intestinal disease severity scores and pVL for all LSI animals with intestinal disease (LSI-Mild and LSI-Severe) and a strong negative correlation between terminal blood $\mathrm{CD}^{+}{ }^{+} \mathrm{T}$-cell counts and severity of intestinal disease in LSI-Severe animals. This indicates that the GI tract is either sensitive to or responsible for dramatic changes in systemic viral replication and associated systemic immunologic dysfunction during chronic infection.
Intestinal disease in animals treated with cART was absent, similar to PC animals, suggesting that cART initiated during acute infection was effective at preventing the development of intestinal disease. This was also true in all gut sites examined. Numerous studies have examined the effectiveness of cART in restoring dysfunction of the gut in HIV-infected people. This has most thoroughly been evaluated by measuring restoration of $\mathrm{CD}^{+} \mathrm{T}$-cell populations in the gut with mixed results. Early intervention, similar to the cART regimen we used, often results in restoration of $\mathrm{CD}^{+} \mathrm{T}$ cells in various intestinal regions; later intervention is typically insufficient. ${ }^{23}$

To better understand alterations to cellular processes and populations in the GI tract, we quantified cells undergoing apoptosis, the numbers of $\mathrm{CD}^{+} \mathrm{T}$ cells and $\mathrm{CD} 68^{+}$macrophages, and the relative numbers of SIV-infected $\mathrm{T}$ cells and macrophages in PC, LSI-Severe, and LSI-None animals. Increased caspase-3 expression and terminal deoxynucleotidyl transferase-mediated dUTP nick-end labeling and reduced tight junction proteins have been detected in chronically HIV-infected humans and SIV-infected macaques. These are associated with a dysfunctional gut barrier. ${ }^{12,26,27}$ In this study, LSI-Severe animals had a significant increase in caspase- 3 immunohistochemical staining in the ileum and trended toward an increase in the colon. These increases coincided with a shift in the location of positively stained cells from the superficial mucosa on villus tips and between colonic glands in PC and LSI-None animals to discrete foci concentrated around degenerating villi 


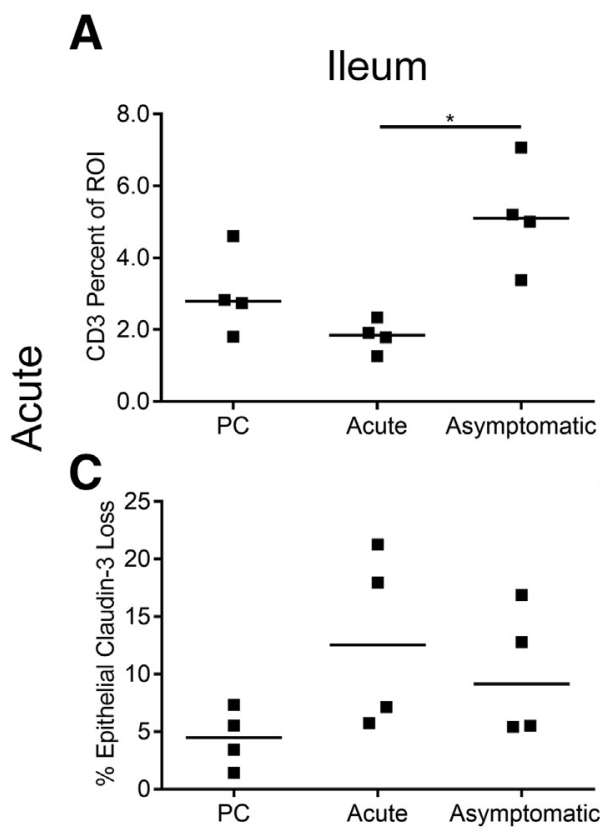

B
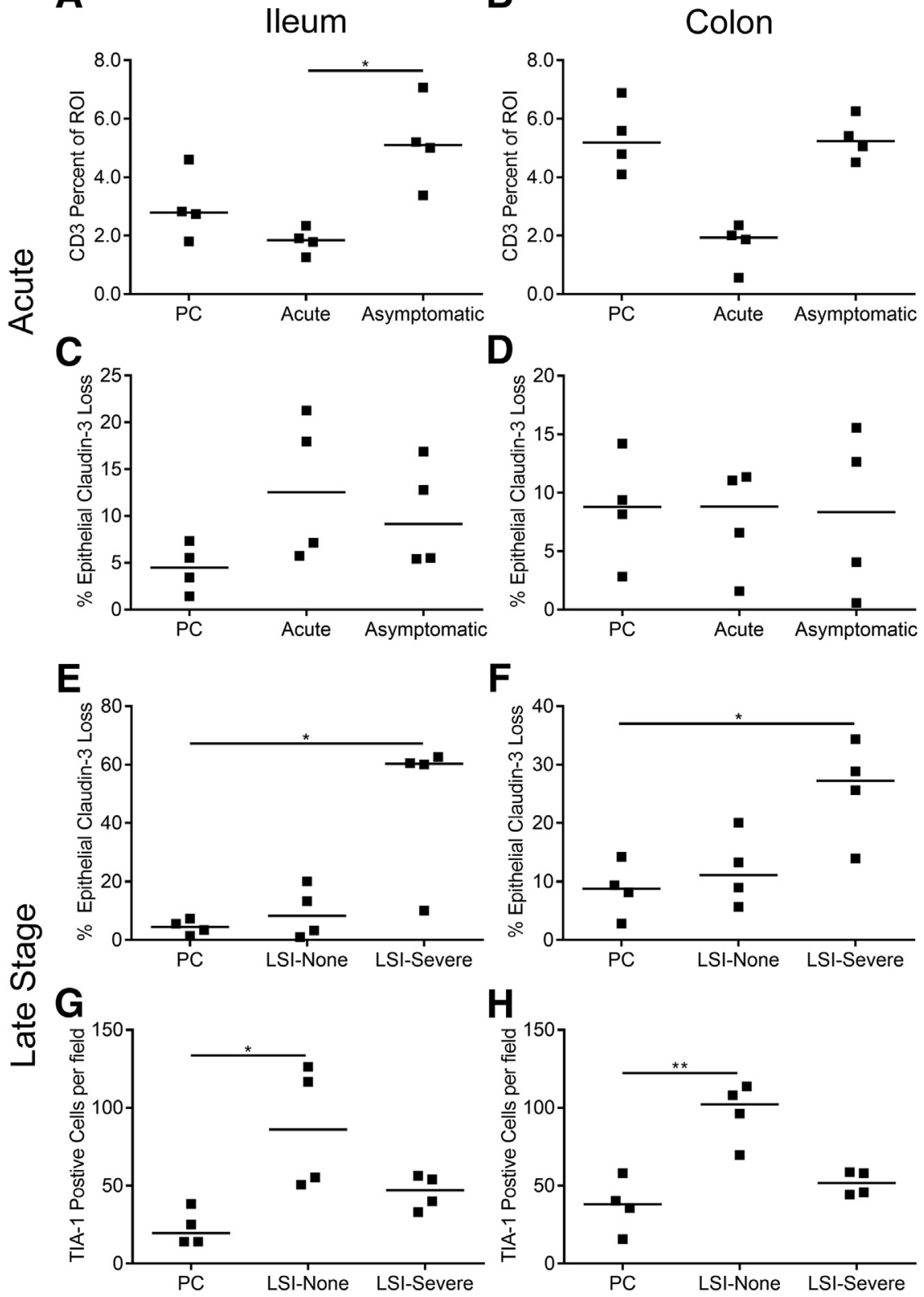

$\mathbf{F}$
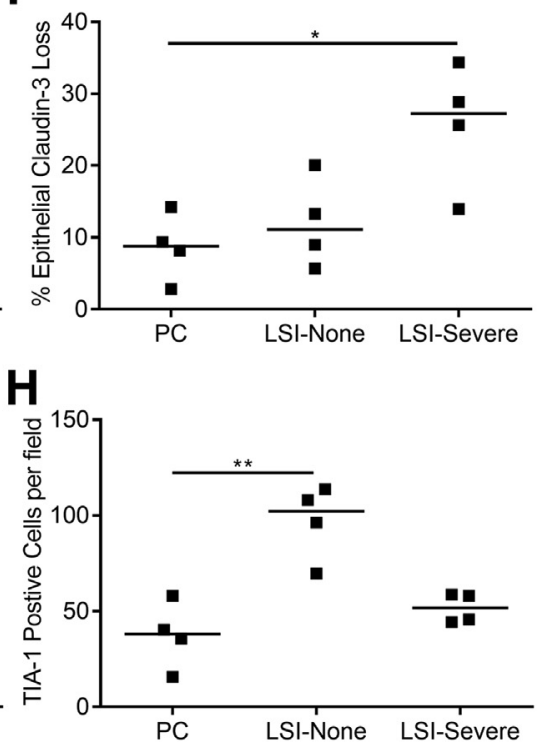

Figure 8 Quantitation of $\mathrm{CD} 3$, claudin-3, and TIA-1 IHC. Percentage of regions of interest (ROIs) positive for CD3 and percentage of epithelial claudin-3 loss by immunohistochemistry during acute and asymptomatic infection (top two rows). Average number of TIA-1-positive cells per field and percentage of epithelial claudin-3 loss by immunohistochemistry in SIV-infected animals at late-stage infection (LSI) with and without severe intestinal disease in the ileum (left column) and colon (right column). A and B: CD3 staining in procedural control $(P C)$, acute, and asymptomatic animals. $\mathbf{C}$ and $\mathbf{D}$ : Claudin-3 staining in PC, acute, and asymptomatic animals. E and F: Claudin-3 staining in PC and LSI animals with or without intestinal disease. $\mathbf{G}$ and $\mathbf{H}$ : TIA-1 staining in PC and LSI animals with or without intestinal disease. Lines at the center of grouped data represent the group median. Exact $P$ values between groups were calculated by Kruskal-Wallis test, followed by Dunn post hoc correction. Acute animals trended toward having less $\mathrm{CD} 3$ staining in the colon than $\mathrm{PC}$ $(P=0.0558)$ and asymptomatic $(P=0.0558)$ animals. ${ }^{*} P<0.05,{ }^{*} * P<0.01$. Acute, day 7 postinfection; Asymptomatic, day 21 postinfection; TIA-1, T-cell intracytoplasmic antigen 1. and glands in LSI-Severe animals. These findings provide experimental support for our histologic observations that villus/glandular disruption may be the most predictive histologic finding of intestinal disease.

It is widely known that the gut is a site of rapid HIV/SIV replication and loss of $\mathrm{CD}^{+}{ }^{+} \mathrm{T}$ cells during acute infection and that the GI tract does not typically recover from this damage. ${ }^{5,6}$ In this study, there was no change to the organization of T cells in the gut between PC and LSI animals; $\mathrm{CD}^{+}$staining was evenly distributed throughout the lamina propria. However, in both the ileum and colon there was a significant and dramatic reduction in the amount of CD3 staining in LSI-Severe animals. These findings agree with observations that T-cell populations are ablated and do not recover after acute infection. However, concentrations of
$\mathrm{T}$ cells in LSI-None animals were similar to those in uninfected animals, demonstrating a previously unreported preservation of $\mathrm{T}$-cell populations in some chronically infected late-stage animals.

Some studies in untreated HIV-infected individuals have reported increased numbers of macrophages in the gut, perhaps due to their relative resistance to lytic HIV infection, and alterations in macrophage phenotype away from an inflammatorily anergic state toward a proinflammatory state in the gut. ${ }^{8,9,24-27}$ In this study, CD $68^{+}$cells in PC animals were found primarily in the periluminal portions of the villi in the ileum and glands in the colon. However, in LSISevere animals large numbers of $\mathrm{CD}^{+} 8^{+}$macrophages were detected surrounding foci of villus and glandular degeneration. In the colon there was a significant reduction 
in the level of CD68 immunostaining in LSI-Severe compared with PC animals. The decrease was found only in the colon, which suggests that different environmental conditions exist in the colon and ileum that result in loss of macrophages in the colon only. These results are distinguished from the typical finding of preservation and even expansion of macrophage populations in the gut during SIV and HIV infection.

The gut is a site of intense viral replication in acutely infected humans and macaques and of ongoing viral replication in the absence of cART. LSI-Severe animals had a significant increase in the number of virus-infected cells in the ileum and a trend toward an increase in the colon compared with LSI-None animals. This increased viral replication was occurring despite tremendous depletion of $\mathrm{T}$ cells in both sites in LSI-Severe animals. There was an increase in the proportion of SIV-infected macrophages in LSI-Severe animals compared with LSI-None animals. Again, this is despite reduction in the numbers of macrophages in the colon of LSI-Severe animals. These findings are unique because we saw low numbers of virus-infected cells in some LSI-None animals despite preservation of T-cell populations and high viral loads in the plasma.

There was a highly significant negative correlation between the number of SIV-positive cells and the level of CD3 staining but not CD68 staining in the ileum of all LSI animals. A similar strong correlation trended toward significance in the colon. There also was a strong negative correlation between $\mathrm{pVL}$ and level of CD3 staining, but not CD68 staining, in the ileum and colon. CD3 also showed a weak, negative correlation with caspase- 3 staining. There was a strong positive correlation between caspase- 3 staining and the number of SIV-infected cells in the ileum and pVL in the colon. Finally, the number of cells positive for SIV in the ileum and colon positively correlated with the amount of virus in the plasma. Note that despite having only 8 samples per comparison these correlations were very strong.

These data taken together with the histologic observations indicate that intestinal disease in this model coincides with increased viral replication, both in the gut and blood, and the subsequent loss of T cells. Nonetheless, only about $50 \%$ of animals with high viral loads and loss of peripheral blood $\mathrm{T}$ cells develop intestinal disease. Further, these data suggest that $\mathrm{T}$ cells and not macrophages are the primary contributors or regulators of virus production in the gut. This T-cell-derived viral pool in the gut has a strong relation with the level of virus in the plasma regardless of the density of intestinal $\mathrm{T}$ cells and level of intestinal disease in the animal. This would indicate that in this model the gut is more reflective of the blood as a viral compartment and not the spleen or brain, which are primary influenced by macrophage infection and inflammation. ${ }^{28,29}$

Indeed, GI tract macrophages arise from a lineage independent of many other tissue sites. They are primarily derived from blood monocytes and not the embryonically seeded progenitors that populate the spleen and brain. ${ }^{30}$
Perhaps late-stage SIV infection in this model captures a physiologic state close to death in which loss of GI tract macrophages in the large intestine may be due to destruction of the seeding pool of blood monocytes and not reflective of direct infection of GI tract macrophages. ${ }^{31}$

Unique to this study is the finding that gut T-cell populations are preserved in LSI in the absence of enteropathy in a large proportion of animals. pVL and terminal CD4 ${ }^{+}$ T-cell counts only correlated with those LSI animals exhibiting intestinal disease, whereas the level of CD3 staining in the gut correlated with pVL for all LSI animals, indicating that intestinal disease is secondary to the health of the gut T-cell population. This agrees with the concept that lack of recovery of $\mathrm{CD} 4$ populations in the gut with cART in humans predicts a poor clinical outcome.

Microbial translocation is a thought to be a common occurrence in chronically HIV-infected individuals and in those on cART, resulting from early destruction of the T-cell populations in the gut and associated epithelial disruption. ${ }^{14-17}$ However, especially in people on cART, the relation between $\mathrm{T}$ cells, intestinal damage, translocation, and inflammation is not clear. We examined several markers of microbial translocation in all groups of animals. Regardless of the assay used, there was no difference between LSI-None and LSI-Severe animals. All LSI animals had significantly increased plasma sCD163, a marker of macrophage activation, and plasma sCD14, a marker of macrophage and monocyte response to lipopolysaccharide, compared with PC animals. In addition, plasma sCD14 was elevated in cART animals despite the absence of any intestinal damage. Plasma LBP was somewhat elevated in LSI animals, although this finding was not statistically significant.

We also examined levels of 16s rDNA in plasma, MLNs, and liver. Plasma levels of $16 \mathrm{~s}$ rDNA were increased in cART animals. Most LSI animals had more plasma 16s rDNA than PC animals but not significantly so. There were no significant increases observed in the MLNs or liver. The sensitivity of 16s rDNA qPCR may be inferior to that of other assays, given the nature of endogenous DNase activity by the host, and 16s rDNA has been shown to be a poorer predictor of translocation and clinical outcome than other assays. $^{32}$

The observation that there were little to no differences in measures of microbial translocation between LSI-None and LSI-Severe animals was unexpected. Most studies indicate that increased intestinal disease and loss of T-cell populations in the gut coincide with increased microbial translocation. In addition, in some human studies and some NHP models this has been reported to occur acutely and set the stage for ongoing inflammation throughout the course of disease. Our data suggest that, although microbial translocation is indeed increased during LSI, it appears to be independent of the severity of intestinal disease. Interestingly, no measurement of microbial translocation correlated with CD3 or CD68 staining or the number of virus-infected 
cells in the gut. Although these findings are surprising compared with untreated chronic HIV or other models of SIV infection, they agree with some of the findings in treated humans in whom acute cART may preserve T-cell populations and result in reduced bacterial burden and a positive clinical outcome. ${ }^{33,34}$

To further elucidate a reason for the apparent lack of microbial translocation in this model we examined animals euthanized at 7 and 21 days postinfection (acute and asymptomatic) despite their lack of intestinal disease. In agreement with most literature on the topic we saw significantly lower numbers of $\mathrm{CD}^{+} \mathrm{T}$ cells in animals 7 days after infection. However, this apparent loss of $\mathrm{T}$ cells during acute infection did not coincide with any increased indicators of intestinal disease via histopathologic examination. Further, T-cell populations at 21 days postinfection had recovered to levels similar to those seen in uninfected animals in the ileum and colon. The recovery of T-cell populations after acute infection, in the absence of cART in this model, differs with conventional thinking on this topic.

Looking beyond overt histologic indications of intestinal disease we sought to evaluate intestinal epithelium integrity directly via the tight junction-associated protein claudin-3. Tight junction complexes are one of several mechanisms that prevent the translocation of microbes across the intestinal mucosa. These complexes respond to inflammation in the gut mucosa ${ }^{35}$ and are directly affected by HIV at least in vitro. ${ }^{36}$ Despite a significant loss of T cells 7 days postinfection we did not see an accompanying loss of epithelial claudin-3 during acute or asymptomatic infection, findings that support the lack of intestinal disease observed via histologic evaluation.

We also evaluated the integrity of the epithelial barrier during LSI whereby some epithelial degradation was observed in some animals on histologic evaluation. Interestingly, animals that exhibited severe intestinal disease had a significant increase in the percentage of loss of claudin-3 staining in the epithelium compared with PC animals. This loss was not observed in animals lacking intestinal disease.

Theorizing about potential drivers of intestinal disease in this model, we sought to quantitate the number of CTLs in the intestinal mucosa. CTLs have the potential to increase epithelial apoptosis through a maladaptive inflammatory response to HIV or other intracellular pathogens and have been implicated in contributing at least in part to epithelial damage during acute HIV infection. ${ }^{33}$ Opposite of our expectations, the number of cells containing TIA-1, a protein associated with the proapoptotic cytoplasmic granules in CTLs, was elevated in LSI-None animals but not in LSISevere animals. Although on the surface counterintuitive, this might suggest that preservation of CTL populations confers a type of intestinal resilience absent in animals that develop intestinal disease. An increase in programmed cell death protein 1 expression on SIV-specific CTLs in the intestines during chronic infection which coincides with functional impairment of adaptive CTL responses has been reported in SIV-infected rhesus macaques that develop intestinal dysfunction and exhibit microbial translocation throughout disease. ${ }^{37}$ Thus, it is possible that in these animals a more robust SIV-specific CTL response may, in part, be responsible for attenuating both intestinal disease and microbial translocation; when these cells are lost, intestinal dysfunction occurs.

A number of potential mechanisms could drive intestinal damage other than CD4 T-cell destruction with functional impairment and loss of virus-specific CTLs. DCs, both plasmacytoid and myeloid DCs, are $\mathrm{CD}^{-} 8^{-}$and may be infected by HIV. Proliferative plasmacytoid DCs have been reported to infiltrate the gut of HIV-infected individuals and up-regulate the proapoptotic enzyme granzyme B. ${ }^{38}$ In addition, proinflammatory mucosal myeloid DCs can be productively infected and are linked to microbial population changes. ${ }^{39}$ These features of inflammation and proliferation in DCs may allow them to contribute to observed enteropathy.

Shifts in the microbiota of the gut have been reported both in chronically infected and cART-treated humans and macaques and are associated with disease progression. Specific taxa are associated with favorable or unfavorable conditions in treated HIV patients. Two examples are increased Proteobacteria and proinflammatory tryptophan metabolism $^{40}$ and increased Lactobacillales and reduced microbial translocation. ${ }^{41}$ Perhaps the predominant type of bacteria that are present and translocating is influencing the gut in unforeseen ways.

Given the rapid progression of disease in this model, it is possible that highly proinflammatory microbial translocation only arises with prolonged intestinal barrier impairment and not acute episodes of increased inflammation. Indeed, our experiments indicate that, at least in this model, the first bouts of microbial translocation are likely to occur during LSI and not in acute infection. This is unique in the body of literature regarding microbial translocation during HIV and SIV infection. This model has been previously shown to recapitulate many aspects of HIV-associated inflammation and immunologic impairment, making these findings regarding translocation all the more important. ${ }^{17-19}$ Indeed, there are likely other mechanisms involved such as viral escape from host control, and further study is warranted.

Our findings highlight the complexities around chronic inflammation in HIV or SIV disease. Collectively, these findings support the need for continued study of the nature of enteropathy in HIV-infected patients through clinical monitoring and SIV/macaque modeling. In addition, we feel there is a discrete need for more rigorous approaches and assays to evaluate the presence and amount of microbial translocation occurring in the host. Ideally, these data along with those from other NHP models of HIV will provide new avenues of therapy and will allow further improvement of the lives of those affected. 


\section{Acknowledgment}

We thank the staff at Johns Hopkins University School of Medicine for the excellent veterinary care.

\section{Supplemental Data}

Supplemental material for this article can be found at http://dx.doi.org/10.1016/j.ajpath.2016.10.019.

\section{References}

1. World Health Organization. Global summary of AIDS epidemic 2015. Geneva, WHO, 2015. Available at http://www.who.int/hiv/data/epi_ core_july2015.png (accessed January 19, 2016)

2. Knox TA, Spiegelman D, Skinner SC, Gorbach S: Diarrhea and abnormalities of gastrointestinal function in a cohort of men and women with HIV infection. Am J Gastroenterol 2000, 95:3482-3489

3. Kotler DP, Gaetz HP, Lange M, Klein EB, Holt PR: Enteropathy associated with the acquired immunodefciency syndrome. Ann Intern Med 1984, 101:421-428

4. Bhaijee F, Subramony C, Tang S-J, Pepper DJ: Human immunodeficiency virus-associated gastrointestinal disease: common endoscopic biopsy diagnoses. Patholog Res Int 2011, 2011:247923

5. Lim SG, Condez A, Lee CA, Johnson MA, Elia C, Poulter LW: Loss of mucosal CD4 lymphocytes is an early feature of HIV infection. Clin Exp Immunol 1993, 92:448-454

6. Brenchley JM, Schacker TW, Ruff LE, Price DA, Taylor JH, Beilman GJ, Nguyen PL, Khoruts A, Larson M, Haase AT, Douek DC: CD4 $+\mathrm{T}$ cell depletion during all stages of HIV disease occurs predominantly in the gastrointestinal tract. J Exp Med 2004, 200:749-759

7. Kanwar B, Favre D, McCune JM: Th17 and regulatory T cells: implications for AIDS pathogenesis. Curr Opin HIV AIDS 2010, 5: 151-157

8. Lim SG, Condez A, Poulter LW: Mucosal macrophage subsets of the gut in HIV: decrease in antigen-presenting cell phenotype. Clin Exp Immunol 1993, 92:442-447

9. Allers K, Fehr M, Conrad K, Epple H-J, Schürmann D, Geelhaar-Karsch A, Schinnerling K, Moos V, Schneider T: Macrophages accumulate in the gut mucosa of untreated HIV-infected patients. J Infect Dis 2013, 209:739-748

10. Lehmann C, Jung N, Förster K, Koch N, Leifeld L, Fischer J, Mauss S, Drebber U, Steffen HM, Romerio F, Fätkenheuer G, Hartmann P: Longitudinal analysis of distribution and function of plasmacytoid dendritic cells in peripheral blood and gut mucosa of HIV infected patients. J Infect Dis 2014, 209:940-949

11. Veazey RS, Rosenzweig M, Shvetz DE, Pauley DR, DeMaria M, Chalifoux LV, Johnson RP, Lackner AA: Characterization of gutassociated lymphoid tissue (GALT) of normal rhesus macaques. Clin Immunol Immunopathol 1997, 82:230-242

12. Klatt NR, Harris LD, Vinton CL, Sung H, Briant JA, Tabb B, Morcock D, McGinty JW, Lifsonm JD, Lafont BA, Martin MA, Levine AD, Estes JD, Brenchley JM: Compromised gastrointestinal integrity in pigtail macaques is associated with increased microbial translocation, immune activation, and IL-17 production in the absence of SIV infection. Mucosal Immunol 2010, 3:387-398

13. Sandler NG, Douek DC: Microbial translocation in HIV infection: causes, consequences and treatment opportunities. Nat Rev Microbiol 2012, 10:655-666

14. Estes JD, Harris LD, Klatt NR, Tabb B, Pittaluga S, Paiardini M, Barclay GR, Smedley J, Pung R, Oliveira KM, Hirsch VM, Silvestri G, Douek DC, Miller CJ, Haase AT, Lifson J, Brenchley JM: Damaged intestinal epithelial integrity linked to microbial translocation in pathogenic simian immunodeficiency virus infections. PLoS Pathog 2010, 6:e1001052

15. Jiang W, Lederman MM, Hunt P, Sieg SF, Haley K, Rodriguez B, Landay A, Martin J, Sinclair E, Asher AI, Deeks SS, Douek DC, Brenchley JM: Plasma levels of bacterial DNA correlate with immune activation and the magnitude of immune restoration in persons with antiretroviral-treated HIV infection. J Infect Dis 2009, 199: $1177-1185$

16. Tincati C, Douek DC, Marchetti G: Gut barrier structure, mucosal immunity and intestinal microbiota in the pathogenesis and treatment of HIV infection. AIDS Res Ther 2016, 13:19

17. Handley SA, Desai C, Zhao G, Droit L, Monaco CL, Schroeder AC, Nkolola JP, Norman ME, Miller AD, Wang D, Barouch DH, Virgin HW: SIV infection-mediated changes in gastrointestinal bacterial microbiome and virome are associated with immunodeficiency and prevented by vaccination. Cell Host Microbe 2016, 19:323-335

18. Clements JE, Mankowski JL, Gama L, Zink MC: The accelerated simian immunodeficiency virus macaque model of human immunodeficiency virus-associated neurological disease: from mechanism to treatment. J Neurovirol 2008, 14:309-317

19. Zink MC, Clements JE: A novel simian immunodeficiency virus model that provides insight into mechanisms of human immunodeficiency virus central nervous system disease. J Neurovirol 2002, 8:42-48

20. Zink MC, Brice AK, Kelly KM, Queen SE, Gama L, Li M, Adams RJ, Bartizal C, Varrone J, Rabi SA, Graham DR, Tarwater PM, Mankowski JL, Clements JE: Simian immunodeficiency virus-infected macaques treated with highly active antiretroviral therapy have reduced central nervous system viral replication and inflammation but persistence of viral DNA. J Infect Dis 2010, 202:161-170

21. Weed MR, Hienz RD, Brady JV, Adams RJ, Mankowski JL, Clements JE, Zink MC: Central nervous system correlates of behavioral deficits following simian immunodeficiency virus infection. J Neurovirol 2003, 9:452-464

22. Zeitz M, Ullrich R, Schneider T, Kewenig S, Hohloch K, Riecken EO: HIV/SIV enteropathy. Ann N Y Acad Sci 1998, 859:139-148

23. Costiniuk CT, Angel JB: Human immunodeficiency virus and the gastrointestinal immune system: does highly active antiretroviral therapy restore gut immunity? Mucosal Immunol 2012, 5:596-604

24. Smythies LE, Sellers M, Clements RR, Mosteller-Barnum M, Meng G, Benjamin WH, Orenstein JM, Smith PD: Human intestinal macrophages display profound inflammatory anergy despite avid phagocytic and bacteriocidal activity. J Clin Invest 2005, 115:66-75

25. Smythies LE, Shen R, Bimczok D, Novak L, Clements RH, Eckhoff DE, Bouchard P, George ME, Hu WK, Dandekar S, Smith PD: Inflammation anergy in human intestinal macrophages is due to Smad-induced I[kappa]B[alpha] expression and NF-[kappa]B inactivation. J Biol Chem 2010, 285:19593-19604

26. Cassol E, Rossouw T, Malfeld S, Mahasha P, Slavik T, Seebregts C, Bond R, du Plessis J, Janssen C, Roskams T, Nevens F, Alfano M, Poli G, van der Merwe SW: CD14(+) macrophages that accumulate in the colon of African AIDS patients express pro-inflammatory cytokines and are responsive to lipopolysaccharide. BMC Infect Dis 2015, $15: 430$

27. Carter CA, Ehrlich LS: Cell biology of HIV-1 infection of macrophages. Annu Rev Microbiol 2008, 62:425-443

28. Williams DW, Engle EL, Shirk EN, Queen SE, Gama L, Mankowski JL, Zink MC, Clements JE: Splenic damage during SIV infection: role of T-cell depletion and macrophage polarization and infection. Am J Pathol 2016, 186:2068-2087

29. Eugenin EA, Clements JE, Zink MC, Berman JW: Human immunodeficiency virus infection of human astrocytes disrupts blood-brain barrier integrity by a gap junction-dependent mechanism. J Neurosci 2011, 31:9456-9465

30. Hoeffel G, Ginhoux F: Ontogeny of tissue-resident macrophages. Front Immunol 2015, 6:486

31. Hasegawa A, Liu H, Ling B, Borda JT, Alvarez X, Sugimoto C, Vinet-Oliphant H, Kim W-K, Williams KC, Ribeiro RM, Lackner AA, 
Veazey RS, Kuroda MJ: The level of monocyte turnover predicts disease progression in the macaque model of AIDS. Blood 2009, 114: 2917-2925

32. Abad-Fernández M, Vallejo A, Hernández-Novoa B, Díaz L, Gutiérrez C, Madrid N, Muñoz MA, Moreno S: Correlation between different methods to measure microbial translocation and its association with immune activation in long-term suppressed HIV-1-infected individuals. J Acquir Immune Defic Syndr 2013, 64:149-153

33. Epple H-J, Allers K, Tröger H, Kühl A, Erben U, Fromm M, Zeitz M, Loddenkemper C, Schulzke J-D, Schneider T: Acute HIV infection induces mucosal infiltration with CD4 + and $\mathrm{CD} 8+\mathrm{T}$ cells, epithelial apoptosis, and a mucosal barrier defect. Gastroenterology 2010, 139: $1289-1300$

34. Epple H-J, Schneider T, Troeger H, Kunkel D, Allers K, Moos V, Amasheh M, Loddenkemper C, Fromm M, Zeitz M, Schulzke J-D: Impairment of the intestinal barrier is evident in untreated but absent in suppressively treated HIV-infected patients. Gut 2009, 58: $220-227$

35. Luettig J, Rosenthal R, Barmeyer C, Schulzke JD: Claudin-2 as a mediator of leaky gut barrier during intestinal inflammation. Tissue Barriers 2015, 3:e977176

36. Nazli A, Chan O, Dobson-Belaire WN, Ouellet M, Tremblay MJ, Gray-Owen SD, Arsenault AL, Kaushic C: Exposure to HIV-1 directly impairs mucosal epithelial barrier integrity allowing microbial translocation. PLoS Pathog 2010, 6:e1000852
37. Xu H, Wang X, Lackner AA, Veazey RS: CD8 down-regulation and functional impairment of SIV-specific cytotoxic $\mathrm{T}$ lymphocytes in lymphoid and mucosal tissues during SIV infection. J Leukoc Biol 2013, 93:943-950

38. Boichuk SV, Khaiboullina SF, Ramazanov BR, Khasanova GR, Ivanovskaya KA, Nizamutdinov EZ, Sharafutdinov MR, Martynova EV, DeMeirleir KL, Hulstaert J, Anokhin VA, Rizvanov AA, Lombardi VC: Gut-associated plasmacytoid dendritic cells display an immature phenotype and upregulated granzyme B in subjects with HIV/AIDS. Front Immunol 2015, 6:485

39. Dillon SM, Lee EJ, Kotter CV, Austin GL, Gianella S, Siewe B, Smith DM, Landay AL, McManus MC, Robertson CE, Frank DN, McCarter MD, Wilson CC: Gut dendritic cell activation links an altered colonic microbiome to mucosal and systemic T-cell activation in untreated HIV-1 infection. Mucosal Immunol 2016, 9:24-37

40. Vujkovic-Cvijin I, Dunham RM, Iwai S, Maher MC, Albright RG, Broadhurst MJ, Hernandez RD, Lederman MM, Huang Y, Somsouk M, Deeks SG, Hunt PW, Lynch SV, McCune JM: Dysbiosis of the gut microbiota is associated with HIV disease progression and tryptophan catabolism. Sci Transl Med 2013, 5:193ra91

41. Pérez-Santiago J, Gianella S, Massanella M, Spina CA, Karris MY, Var SR, Patel D, Jordan PS, Young JA, Little SJ, Richman DD, Smith DM: Gut Lactobacillales are associated with higher CD4 and less microbial translocation during HIV infection. AIDS 2013, 27: 1921-1931 